\title{
Simplified Framework for Benchmarking Standard Downlink Scheduler over Long Term Evolution
}

\author{
Srinivasa $\mathrm{R} \mathrm{K}^{1}$ \\ Research Scholar, Visvesvaraya Technological University \\ Department of Electronics and Communication Engineering \\ BIT, Bangalore, India
}

\begin{abstract}
Downlink scheduling is one of the essential operations when it comes to improving the quality of service in Long Term Evolution (LTE). With an increasing user base, there will be an extensive challenge in resource provisioning too. A review of existing approaches shows that there is a significant possibility of improvement in this regard, and hence, the proposed manuscript presents a benchmarking model for addressing the issues associated with Best-Channel Quality Indicator (CQI), Round Robin, and Hybrid Automatic Repeat Request (HARQ). The outcome shows HARQ scheduling to offer better performance in higher throughput, higher fairness, and lower delay over different test cases.
\end{abstract} Robin

Keywords-eNodeB; Scheduler; HARQ; Best-CQI; Round

\section{INTRODUCTION}

The user-base of mobile communication is increasing rapidly, especially due to internet accessibility with the devices. The internet's capacity for these devices provides a base or a platform to build many applications that can use high dimension data like multimedia, including audio, image, and videos, but these applications are bandwidth-hungry. It demands a very high data transfer rate. The exploration of the finding possibilities of the usage of the spectrum in an optimal ways leads the new standardization of the architecture and communication process to meet the goal of providing adequate resources to the applications, and that shows the evolution of a new generation or era of the wireless communication systems [1]. In every generation of the standard, the data services maximize the high-speed packet access method to achieve a higher data rate, low latency, which is a goal of Long-Term Evolution (LTE) to support a very high demand of the traffic, which aim to evolve the universal terrestrial access network [2].

The typical LTE standard provisions the specifications, as mentioned in Table I. Due to the use of the frequency and time division duplexing, MIMO, and OFDM [3]. In the OFDM, the time-frequency grids are divided into the number of the resource blocks (RB) symbols, and a scheduler operation assigns or allocates the RBs to the users. Every entry in the $\mathrm{RBs}$ is known as a resource element (RE). There are two states of the art scheduling algorithm; one is the round-robin scheduling algorithm (RRSA), where every user gets equal privileged ignoring the indications of the quality of the channel, whereas in the second method, namely, best channel quality index(B-CQI), where the users are allocated the RBs

\author{
Dr. Hemanth Kumar A. ${ }^{2}$ \\ Professor and Principal Investigator (R\&D) \\ Department of Electronics and Communication Engineering \\ BIT, Bangalore, India
}

only if it has superior channel quality index. The physical layer characteristics, modulation scheme, and the coding process influence the scheduling algorithm's design [4].

A closer look into the literature shows that there have been various dedicated research attempts towards addressing downlink scheduling issues in LTE. But it is noticed that existing approaches do not meet the demands of the quality-ofservice performance that is required in the practical world scenario. It is also noticed that monitoring of the state of memory associated with the end-users is also considered to address this downlink scheduling problem. Still, such an approach doesn't consider various factors that are needed to meet the practical world application. At present, there are more dedicated attempts towards downlink scheduling issues focusing on an advanced version of LTE, i.e., $5 \mathrm{G}$ networks, i.e. [5][6][7]. This acts as positive motivation towards carrying out the current work in the proposed system.

The proposed manuscript evaluates two state-of-the-art scheduling algorithms: the best channel quality index (B-CQI) and the round-robin to benchmark its performance with the proposed HARQ-Scheduler as HARQ-S. The contribution of the proposed system is as follows: i) to present a design of novel scheduler using best channel quality indicator, ii) to include investigation using round-robin scheduler and HARQ based scheduler, iii) to develop a localization process for specific users over the base station of the user, iv) to carry out a benchmarking testbed for assessing the potential in downlink scheduling. The organization of the paper is as follows: Section II discusses LTE characteristics followed up by the research trend in Section III. Discussion of standard schedulers subjected to benchmarking is carried out in Section IV, while result discussion of the proposed study is carried out in Section V, while the summary of the proposed study is written in Section VI as a conclusion.

TABLE I. LTE STANDARD PROVISIONS THE SPECIFICATIONS

\begin{tabular}{|l|l|}
\hline $\begin{array}{l}\text { Downlink peak data } \\
\text { rate }\end{array}$ & $300 \mathrm{Mbps}$ \\
\hline Uplink peak data rate & $75 \mathrm{Mbps}$ \\
\hline Latency & $\geq 5 \mathrm{~ms}$ \\
\hline Bandwidth Range & $1.4 \mathrm{MHz}$ to $20 \mathrm{MHz}$ \\
\hline MIMO + OFDM & Improves throughput and optimal spectrum sensing \\
\hline
\end{tabular}




\section{LTE CHARACTERISTICS AND ITS INFLUENCE ON THE DESIGN OF SCHEDULER}

The LTE and LTE-A and the future evolution of communication standards keep a common goal of optimal spectrum utilization and offer a very high data rate with low latency in lower operating costs, a multi-functional optimization problem.

\section{A. LTE General Architectural Characteristics}

LTE's core building blocks collaboratively work to achieve approximately $10 \mathrm{Mbps}$ and $50 \mathrm{Mbps}$ data rates in downlink and uplink, respectively. The LTE system involves minimal network connections to minimize the latency between User Equipment (UE) and control planes by introducing a mobility management scheme (MMS) that ensures lower operational cost. In LTE, the bandwidth range is between $1.4 \mathrm{MHz}$ to 20 $\mathrm{MHz}$, which operates in pairing and non-pairing of the spectrum to support both time and frequency duplexing, which are TDD and FDD.

LTE adopts spatial multiplexing and multiple antennas to improvise the signal power on either side of the transmitter and antenna as the signal power $\left(\mathrm{P}_{\mathrm{s}}\right)$. The data rate $(\mathrm{D})$ follows the relation as per eq. (1).

$P_{S} \propto D \ldots$

The LTE system utilizes all the previous standard spectrum frequency bands (SFB) and additional SFB, making LTE more robust and interoperable. Though the basic architecture of LTE is designed to achieve a coverage of approximately 90 to 100 $\mathrm{Km}$, whereas, at $33 \%$ or higher coverage, it suffers degradation into the network's efficiency.

Fig. 1 illustrates a basic architectural building block of an LTE system, where the evolved E-UTRAN collaborates many numbers of eNodeB and evolved packet core (EPC) supported with OFDM, MMS, FDD, and TDD. The system attains a better data rate at a lower UE speed. The effective scheduling algorithm requires considering these parameters and facts along with the physical layer attributes. Section B below describes the physical layer's essential aspects that impact the effective scheduling algorithm's LTE design aspect.

\section{B. Physical Layer Characteristics}

Seamless roaming operation is achieved in LTE by integrating the previous generation frequency band and new frequency bands. The duplex mode FDD operates in 25 combinations of operating frequencies for uplink and downlink in the paired manner. In contrast, TDD's duplex mode uses in 9 varieties of operating frequency band common for uplink and downlink [8]. Both unicast and multimedia broadcast multiple services (BMS) improvises the signal-to-noise ratio (SNR) [9]. The channel bandwidth ranges from $1.4 \mathrm{MHz}$ to $20 \mathrm{MHz}$ with varied resource block size ranging in the slots of $\{6,15,25,50$, $75,100\}$.

\section{Technologies and Process of Packet Scheduler in LTE}

The supporting technologies such as OFDM, MIMO, and Channel coding, adaptive-link combined functions to fulfill LTE requirements to a greater extent with ease of transmission bandwidth allocation and additional resource optimization using overcoming multipath fading, mapping resource elements with OFDM signal.

The forward error correction (FEC) and Automatic repeat request (ARQ) handle the transmitted data errors. The link adaption is performed using adaptive modulation and coding (AMC); the modulation schemes are decided based on signal to interference noise ratio (SINR). The generation of a signal in the LTE physical layer using appropriate coding and modulation is shown in the Fig. 2.

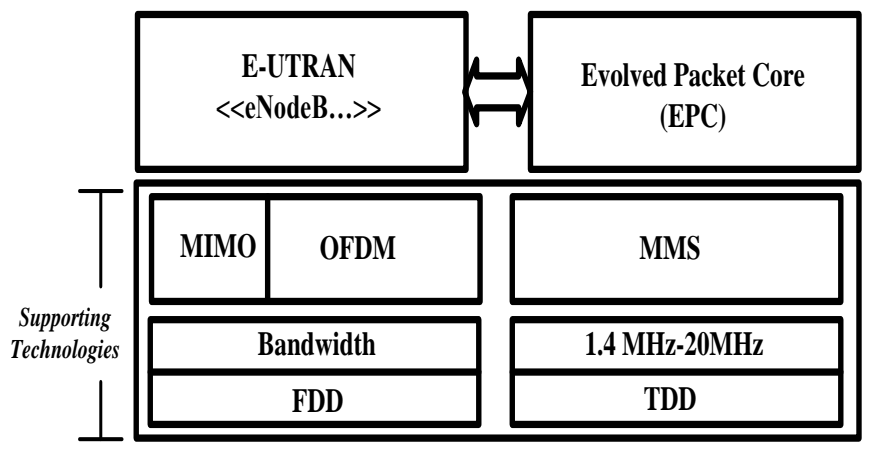

Fig. 1. Architectural backbone of an LTE system

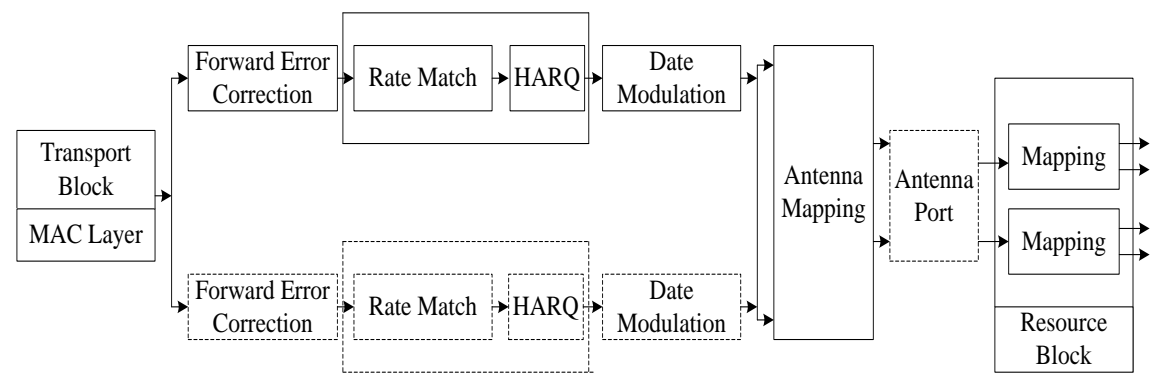

Fig. 2. OFDM Modulated Signal Generation Process. 
The mapping of appropriate coding and modulation schemes correlates with channel quality indicator (CQI) measured at UE. The channel quality (CQ): $\rightarrow\{S, C, Q R\}$, where $\mathrm{S}=\mathrm{SINR}, \mathrm{C}=\mathrm{Channel}$, and $\mathrm{Q}_{\mathrm{R}}=$ Receiver's rate. The channel or the signal quality is represented by reference symbols and has its placement mechanism in the resource blocks. In this manner, the appropriate resource block and the time slot decision take place based on the value of CQI in a periodic and aperiodic manner using physical uplink control channel (PUCCH) and physical uplink shared channel (PUSCH), respectively. The computation of the number of subbands $\left(\mathrm{N}_{\mathrm{sb}}\right)$ takes place using eq. (4), where the total bandwidth (B) is the total sum of $\mathrm{N}_{\mathrm{sb}}$ sub-band as per eq. (2).

$B=\sum_{i=1}^{N}\left(N_{s b}\right) i$

Such that $\left(N_{s b}\right)_{i}=\left\{M_{R B}\right\}_{j}$, where 'j' = index of resource blocks and the number of the resource block $\left(M_{R B}\right)$ in $\mathrm{B}$, with downlink (DL), is computed using eq. (3).

$\left(N_{S b}\right)^{D L / M_{R B}}$

Therefore, $\left(N_{s b}\right)=\left(N_{s b}\right)^{D L / M_{R B}} /\left(M_{R B}\right)$

The core component of traffic management takes place using packet scheduler positions placed in the base station (BS), which is responsible for allocating resources, especially spectrum, to the UE depending upon the channel conditions. There is a trade-off between maximizing the efficiency of spectrum or resources through a useful scheduler or resource allocator that degrades the network's performance by minimizing throughput and fairness. Therefore, the scheduling algorithm's effective design approach considers the intrinsic correlation between CQI, UE data buffer status, and the requirement of the quality of service to map the RBs with appropriate selection of coding and modulation schemes.

\section{RELATED WORK}

Generally, the delay profile represents more than one version of the transmitted signal at the receiver side. The authors Hani and Samota [10] improvises the base model of the LTE by mapping it to the Rayleigh Fading model for a delay profile in the urban scenario to improve the Delay while accessing the Physical Random-Access Channel (PRACH) using a combined approach of the scheduling and particle swarm optimization. This Model exhibits an approximately 13$14 \%$ reduction in the Delay even if the Round Robin Scheduling is not used.

The higher throughput for both uplink and downlink is an essential requirement for better QoS in modern communication systems, which are shared by the multi-users. In contrast, there exists a contradiction of the resource allocation process between pooling and severing as requested. The advanced UMTS Terrestrial Radio Access Network (UTRAN) in LTE facilitates a wide range of scheduling mechanisms to offer users resources. The authors Saxena et al. [11] has performed a critical analysis of the performance metrics, including a) throughput, b) spectral efficiency, and c) fairness for the various schedulers that includes: a) resource fair, b) round- robin, c) best channel indicator (CQI). It is observed that though the users get more resources, overall network performance degrades.

The authors Minelli et al. [12] evaluates proportional fair (PF) and round-robin (RR) using statistical throughput evaluation model for the context of relay-enhanced LTEA and suggest they are not appropriate methods as both the relay nodes and the backhaul link quality influences the choice of the Scheduler.

The higher data rate demand continues right from the $2 \mathrm{G}$ with the introduction of data services, which was improvised in $3 \mathrm{G}$ with HSPA and LTE in 4G. The inclusion of OFDM enhances the throughput, but the effective scheduling process can improvise the overall performance. The downlink scheduling plays an important role as the higher data rate from the base station to the user equipment ensures low packet loss. An extensive comparison of downlink scheduling is performed by the authors Ramesh [13], and they list popular algorithms like a) Exponential Rule, b) Proportional Fair, c) Round Robin, etc.

The high data rate in LTE or new radio (NR) for the multimedia applications is achieved through the downlink scheduling like Proportional Fair, round Robin, or by the best Channel Quality. These scheduling approaches have a major limitation: the MAC requires making decisions during every transmission time interval for the allocation of resources for UEs, which is an overhead if there is no change in the channel condition or data scheduling, which increases the processing time overhead. The authors Chilmulwar and Sinha [14] introduces a MAC scheduler using a machine learning approach of namely Autoregressive Integrated Moving Average, that minimizes the resource use of $\mathrm{PDCCH}$ along with the time required for the resource allocation to the UE.

In many of the approaches, in the downlink throughput analysis considers a uniform distribution of UEs in the cell, whereas if the proper modeling takes place for this analysis, then many of the effects like spatial distribution of UEs, BS, and network functions can be exploited which affect the throughputs. The authors Olaifa and Arifler [15] use a simulator, namely Vienna, to quantify the throughput analysis and found that if the UEs are in the group near the BS exhibit varied throughput as compared to the uniformly deployed UEs. In real-time strategy, the BS is deployed newer to the dense user bases. Another significant observation was made that the proportional Fair is less advantageous than that of the roundrobin in the case of grouped or clustered users surrounding the BS.

The inclusion of the Carrier Aggregation enhances the throughput; therefore, the CA enables down line schedule for eNodeB to ensure higher QoS, but at the same time, the aggregation poses power overhead. The authors Chaudhuri and Das [16] claims a first of its kind of download scheduler, which considers QoS, Resource block, and carrier power. Their algorithm gains twice the throughput in the one hundred cell UEs scenario than the round-robin, Efficient Packet Scheduling, and others. 
Thought the objective of the useful Scheduler is to provide better QoS and QoE to UEs. In contrast, the performance is also correlated with the traffic dynamic and resource allocation mechanism. The effect of the TCP variance and combination of the Scheduler on the performance is studied by the authors Adesh and Renuka [17] and observe that the TCP-Westwood performs best out of all combinations. The authors Sundari et al. [18] implement a scheduler to improvise its performance, where they use service class for the bandwidth and resource allocation dynamically with the channel into consideration and offers better understanding as compared to the Round Robin (RR), Best Channel Quality Indicator (BCQI), Proportional Fair (PF) and Opportunistic scheduling. The research of minimizing the energy consumption by the eNodeB is an open research problem in LTE, In the work of Rebekka et al. [19] utilizes the spectrum effectively in reduced energy using the suitably allocate the resource blocks so that even throughput improvises. The Model is benchmarked with the Round robin and Best CQI in terms of the performance metrics like throughput and energy consumption, and fairness.

Yildiz and Sokullu [20] provide consistency in throughput and fairness in the change's mobility pattern compared to the round-robin and best CQI. Another benchmarking by Hayuwidya, Ernawan, and Iskandar [21] uses Monte Carlo simulation and a radio planning software, namely Atoll, and found that proportional fair algorithm for scheduling outperforms as compared to the round-robin. The LTE-sim is used to study the trade-off between the throughput and the fairness of Scheduler Round robin, Maximum throughput, etc., in realistic traffic conditions. Ahmed and AlMuhallabi [22]. The traditional scheduler, including a,) Proportional Fair, b) Best -CQI and c) Round Robin, lack to meet the real provisioning of the optimal throughput and latency. The authors Kayali et al. [23] formulate the scheduling problem as an optimization problem with the inclusion of the constraints of deadline time for the various packets, a minimum buffer of the $\mathrm{UE}$, and develops an objective function to minimize the packet loss and evaluates their Model with LTE-Sim. The regular scheduler performance is assessed in macro-femtocells Hajjawi et al. [24]. A fair boundary scheduling algorithm is proposed by Rahman et al. [25] to handle the trade-off between throughput and fairness and better fairness concerning the round-robin and Proportional Fair.

The authors Sundari et al. [26] proposes a dynamic multitraffic scheduler to allocate the bandwidth and the resources dynamically by considering the UE channel status and performs better than a round-robin and Best Chanel quality indicator. The authors Alotaibi and Akl [27] also propose a packet scheduler where both the UE and the physical resource block are considered design parameters and perform better than round-robin. The authors Harkusha et al. [28] proposes a method of frequency and schedule and the security and optimization of the bandwidth allocation to UEs. It is generally observed that the Round-robin balances the fairness issue, whereas the Max Signal to Noise ratio scheduler maximizes the throughput Dadi and Chibani [29]. The performance of the Scheduler gets influence based on the UE mobility as well its density in a cell. Shams et al. [30] have compared the popular round robin and the proportional Fair in the HetNet to ascertain which Scheduler is appropriate in case of very high mobility. The summary of the essential literature from the above discussion is now tabulated in Table II.

TABLE II. SUMMARY OF THE ABOVE-DISCUSSED STUDIES

\begin{tabular}{|c|c|c|c|}
\hline Cite No & Context & Solution approach & Performance \\
\hline Hani et al(2018)[10] & Urban Delay profile & Scheduling, PSO & $\begin{array}{l}13-14 \% \text { delay reduction without round- } \\
\text { robin }\end{array}$ \\
\hline Saxena et al(2016)[11] & UTRAN and LTE & $\begin{array}{l}\text { Performance evaluation of many scheduling } \\
\text { algorithms }\end{array}$ & $\begin{array}{l}\text { Resource provision improvises and } \\
\text { degrades the network performance }\end{array}$ \\
\hline Minelli et al. (2016)[12] & relay-enhanced LTE-A networks & $\begin{array}{l}\text { Performance proportional fair }(\mathrm{PF}) \text { and } \\
\text { round-robin (RR }\end{array}$ & $\begin{array}{l}\text { statistical throughput evaluation model } \\
\text { indicates not a suitable approach }\end{array}$ \\
\hline Ramesh et al. (2019)[13] & Downlink scheduling in LTE & $\begin{array}{l}\text { Exponential Rule, b) Proportional Fair, c) } \\
\text { Round Robin, etc }\end{array}$ & Performance analysis \\
\hline $\begin{array}{l}\text { Chilmulwar et al. } \\
\text { (2019)[14] }\end{array}$ & MAC scheduling & Machine Learning & $\begin{array}{l}\text { Reduces the time overhead for resource } \\
\text { allocation to UEs }\end{array}$ \\
\hline Olaifa, 2016 [15] & Clustered UE near BS & Vienna Simulator for throughput analysis & $\begin{array}{l}\text { The proportional Fair provides inferior to } \\
\text { Round robin }\end{array}$ \\
\hline Chaudhuri, 2016[16] & CA-based Scheduler & $\mathrm{RB}$, Carrier power, QoS in Scheduler design & $\begin{array}{l}\text { Provides double throughput as compared to } \\
\text { the RR, EPS }\end{array}$ \\
\hline Adesh et al., 2017[17] & Traffic Dynamic & TCP variance with schedulers effect & $\begin{array}{l}\text { The TCP Westwood performs best with all } \\
\text { the schedulers. }\end{array}$ \\
\hline Sundari, 2015, [18] & Service Class & Dynamic bandwidth and resource allocation & $\begin{array}{l}\text { Improvises the performance with traditional } \\
\text { schedulers }\end{array}$ \\
\hline Rebekka, 2015,[19] & eNodeB & Optimal resource block allocation & $\begin{array}{l}\text { Improves the throughput with lesser energy } \\
\text { use. }\end{array}$ \\
\hline Yildiz, 2017 [20] & LTE-A with Mobility & Mobility aware downlink scheduling & Robust to mobility \\
\hline Hayuwidya, 2017[21] & Release 8 LTE & MCS and Atoll 3.2.1 & Proportional Fair outperforms \\
\hline Ahmed,2016[22] & Realistic LTE Traffic condition & LTE-Sim & $\begin{array}{l}\text { The trade-off between throughput and } \\
\text { fairness }\end{array}$ \\
\hline Kayali,2017[23] & LTE & Optimization and LTE-Sim & The decrease in packet loss \\
\hline Hajjawi,2016[24] & Macro-femtocells over the LTE-A & Effect of Scheduler on Congestion & Minimizes cell congestion in LTE \\
\hline Rahman et al., 2016[25] & Trade-off fairness and throughput & Fair Boundary & Better fairness \\
\hline
\end{tabular}




\section{State of the ART Scheduling AlgorithM}

In each time interval, the Scheduler controls the frequency and time resource allocations and the allotment of the resource blocks (RBs), which takes place accordingly, whereas achieving optimal throughput and fairness is an open research problem. The proposed framework evaluates the two popular schedulers, namely Best-CQI and the Round-robin, with the proposed HARQ based Scheduler.

\section{A. Best Channel Quality Indicator Scheduler (BCQI-S)}

The eNodeB receives the CQI by the UEs, and the eNodeB, which acts as a base station, sends the reference signal to the UEs, and accordingly CQI based computation of RBs takes place. The UEs with the best CQI get the RBs, whereas the far distant users do not get RBs as their radio links are inferior as a result provides higher throughput and lower fairness.

\section{B. Round Robin Scheduler}

This algorithm does not consider the channel quality, and it allocates RBs to the UEs according to the availability of the RBs on an FCFS basis. As a result, it provides better fairness and lower throughput.

\section{HARQ Based Scheduler}

In both the BCQI-S and RR-S, the packet error overhead is not predominantly considered, which provides inferior performance in the case of very noisy channels. In contrast, LTE's very objective of achieving a high data rate in the robust condition requires a high data rate transfer in a very fast and reliable manner. Therefore, both the eNodeB and the UE adopt the resource block scheduling techniques and the packet error detection and correction mechanism using either ARQ or HARQ.

In HARQ, the packet detection and correction are generally implemented at the PHY layers, and the control mechanism on the MAC layer tries to correct the packets. Some acceptable erroneous packets are forwarded to the upper layer using its buffer mechanism using a hybrid capacity of a forward error correction (FEC) and automatic repeat request (ARR). In ARQ, it discards the packet if it is erroneous. The future generation applications are multimedia data-hungry, where very high throughput, fairness, and low latency only can ensure higher QoS and, finally, better QoE. This is an open research problem to design a schedule that maximizes both the throughput and the fairness to achieve higher network performance. Therefore, the Proposed HARQ-S aims to optimize both the throughput and fairness even in highly noisy channel conditions.

In the proposed HARQ-S, the allocation of the RBs takes place in two ways, in one slot period, $\mathrm{RBs}$ are allocated only if it justifies the desired CQI and in another slot based on request priority. The erroneous packets are handled by the FEC and ARR using a buffering technique to handle the erroneous packets. The buffer complements the erroneous packets and continues the NACK till the packet is complete; an ACK is received, as shown in Fig. 3.

The system model-independent variable of HARQ-S is listed in Table III.

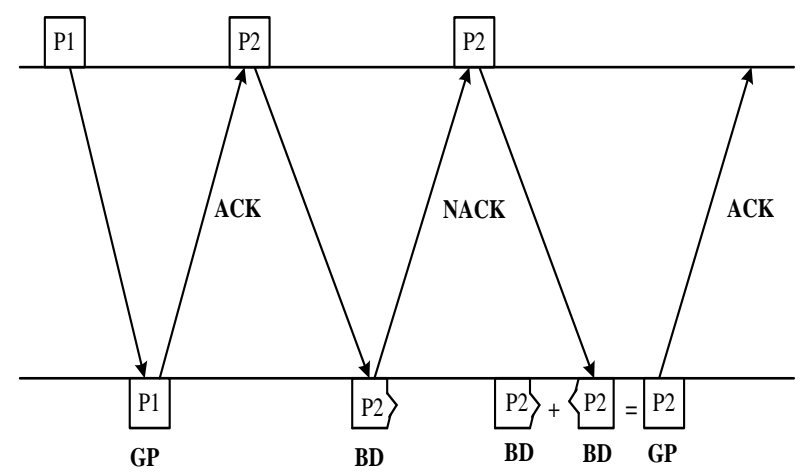

Fig. 3. Packet Error Correction Mechanism in HARQ.

TABLE III. INDEPENDENT VARIABLE OF THE MODEL

\begin{tabular}{|l|l|l|l|}
\hline Sl. No & System Variable & Symbol & Range \\
\hline 1 & Number of user's equipment & nUE & $25,75, \ldots 225$ \\
\hline 2 & Number of Resource Block & nRB & \\
\hline 3 & Time Slots & Ts & \\
\hline 4 & System Bandwidth & B & \\
\hline 5 & Transmit Power & P & \\
\hline 6 & HARQ-Type & HT & \\
\hline
\end{tabular}

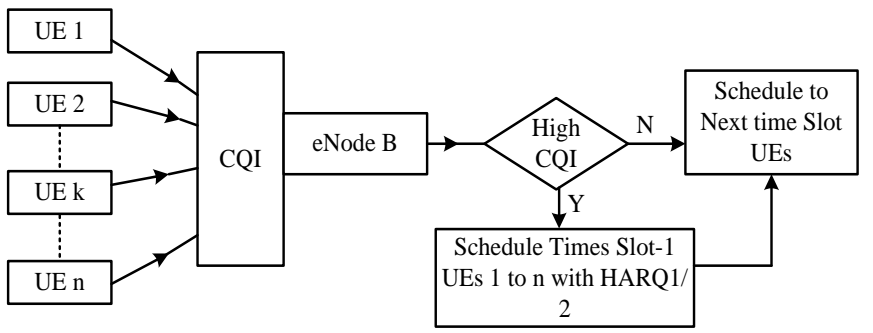

Fig. 4. Process flow of the HARQ-S.

Where the RBs are allotted on the combined basis of CQI, HARQ, and priority.

\section{HARQ-S Analytical System Modelling}

The designed mathematical Model to imitate the behavior of the scheduling algorithm considers a system with a uni-base station (U-BS) with proximity uniformly with the random deployment of the user's equipment's (UE) in such a way that the localization of the U-BS takes place in the centrality of the cell zone of the dimension of 'L' $\mathrm{x}$ 'B' units of the length. The evaluation parameters initialized are listed in Table IV.

TABLE IV. INITIAL PARAMETERS AT THE NODE DEPLOYMENTS

\begin{tabular}{|l|l|l|l|}
\hline Sl. No & Parameters & Symbols & Initialization \\
\hline 1 & No. of Antenna @ U_BS & An & 2 \\
\hline 2 & No. of Antenna @ UE & Au & 1 \\
\hline 3 & System Bandwidth & Bw & $1.4 \mathrm{MHz}$ \\
\hline 4 & System Noise & Sn & $10 \mathrm{~dB}$ \\
\hline 5 & Noise Spectral Density per Hz & Sdn & $174 \mathrm{dBm}$ \\
\hline 6 & Transmit Power at U-BS & Pt & $0 \mathrm{dBm}$ \\
\hline
\end{tabular}

The process flow of the HARQ-S is shown in Fig. 4. 
1) Localization of Kth User and Positioning of U-BS: The localization of all the UEs falls randomly within the proximity of the deployment's region of $\mathrm{L} \times \mathrm{B}$ in such a way that a uniform random placement takes place within the boundary of the deployment location as $\mathrm{UE}_{\mathrm{x}} \& \mathrm{UE}_{\mathrm{y}}$ using equation (5).

$U E_{x} \& U E_{y}=\sum_{k=1}^{U E_{t o t}} f\left(U_{k}\right)$

Further, with the Euclidian distance computation, the localization vector ' $\mathrm{L}$ ' is computed for all the user equipment using the equation (6).

$D U E_{i j}=\sqrt{\sum\left[\left(U i j x^{2}\right),\left(U i j y^{2}\right)\right]}$

The designed concept of HARQ-S evaluates the CQI measures from the UE with two-way slots measurements. According to the justifiable CQI from UE, the primary allocation of RB to the UE in slot- 1 takes place. On the other hand, in slot-2, the RB allocation considers the UE's priority and its associative packets. The system evaluates both HARQ1/HARQ2 to deal with erroneous data packets regardless of the channel conditions. A code is utilized for instantaneous error detection and correction from the data packets (pkt) received in this design context. If the code can correct the erroneously received packet (pkt), it will check and validate the (pkt); else, it will provide NACK and discard the packet, and again it will ask for re-transmission. When the retransmission process occurs and the retransmitted pkt is received at the receiver side, the receiver performs decoding. With an unsuccessful attempt again, the pkt error is encountered. The entire process gets repeated till the system receives successful acknowledgment of the correct pkt receipt. However, this process is quite lengthy due to more parity check bits' computation during the code's error detection and correction. The system optimizes the HARQ1 with scheduling time-slot 1 and time-slot2. And in this case, the FEC plays a very significant role in error correction, even if in the presence of bad channel conditions. HARQ1 design accomplishes a higher throughput curve as compared to the traditional ARQ scheme. Fig. 5 exhibits the deployment architecture of the eNodeB with UEs in the formulated HARQ-S based downlink scheduling concept for LTE.

The system also prioritizes HARQ2 for pkt scheduling and promotes delay-sensitive low-queuing to maximize the effective user throughput with fairness index. In the HARQ2 scheme also the re-transmission process is carried out with the same information. The pkts are also encoded with the same channel codes, and the carrier signal comprising the encoded bits is further subjected for decoding with the maximum-ratiocombining (MRC) algorithm. Here the combination process takes place before the decoding process and just after the demodulation. The process of HARQ does not pose any additional redundant bits during the time of re-transmissions.

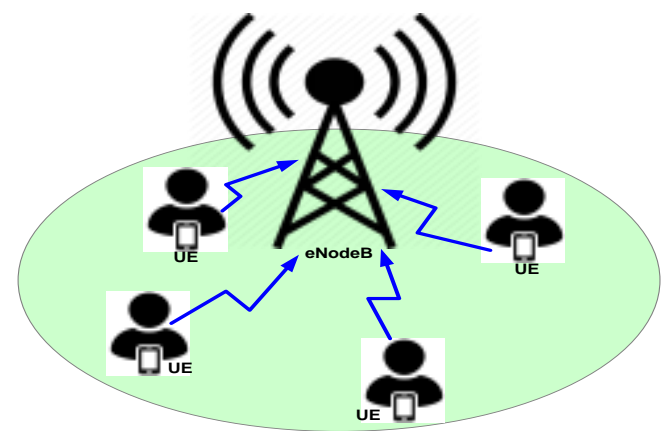

Fig. 5. Deployment of the eNodeB and UEs.

The system employs a flat-architecture of LTE system comprising eNodeBs, which communicates with the core network's respective UEs. The assumption for setting up the channel status metric considers that the smallest RB comprises of $N_{s b}=12$ where the $B$ is considered $180 \mathrm{kHz}$ with $1 \mathrm{~ms}$ of duration. The system designs the formulated HARQ-S based packet scheduling for LTE downlink transmission in a way that aims to attain a higher data-rate and fairness to the UEs, even if in the presence of an erroneous channel. The HARQ-S also targets multi-UEs diversity by mechanizing modulation and coding scheme (MCS), which indicates the channel quality and is frequently reported by the UEs.

The HARQ-S based scheduling algorithm for downlink considers transport block (TB) entity which carries information pkt with headers (h) by interacting variant protocol layers. The MCS associated with RB for each UE influences TB's size and depends on the size of the pkt, which is supposed to be transmitted to the user.

The HARQ-S based pkt scheduling schema considers the UEs at different Ts. The total computation of nUE can be performed with the following mathematical expressions.

$n U E=\operatorname{ArgMax}\left\{\phi(i) \times p k t_{\text {delay }}(T S)_{U E(i)} \times \frac{T_{\text {bits }}(D L)_{U E(i)}}{A v g_{\text {Throughput }} U E(i)}\right.$

Here $\phi(i)$ denotes a maximum probabilistic factor of $p k t_{\text {delay }}(T S)_{U E(i)}$ the head of the line (HOL) pkt delay factor for user i UE(i). and also, $T_{b i t s}(D L)_{U E(i)}$ here indicates the cumulative number of bits for pkt required for DL transmission. And $A v g_{\text {Throughput }} U E(i)$ represents the average throughput for $\mathrm{i}^{\text {th }} \mathrm{UE}$ at Ts slot-1 or slot-2. Here the problem formulation is derived based on selecting the nUE at each Ts to achieve maximum throughput with low-latency of pkt transmission while satisfying the $\mathrm{f}_{\mathrm{i}}$. The problem formulation of the proposed approach is considered as follows from the optimization viewpoint. 
To Maximize $\rightarrow n U E$ with fairness index $\left(\mathbf{f}_{\mathrm{i}}\right)$

Subjected to $A v g_{\text {Throughput }} U E(i)$ within buffered lesser Delay $\left(b_{\mathrm{d}}\right)$

Problem condition-1: Subjected to $n U E$ :

$\operatorname{ArgMax}\left\{\phi(i) \times p k t_{\text {delay }}(T S)_{U E(i)} \times \frac{T_{\text {bits }}(D L)_{U E(i)}}{A v g_{\text {Throughput }} U E(i)}\right.$

when $\mathrm{i} \leftarrow$ non-HARQ users and $\mathrm{p}(\mathrm{T})>T_{b i t s}(D L)_{U E(i)}$

Problem condition-2: Subjected to $n U E$ :

$\operatorname{ArgMax}\left\{\phi(i) \times p k t_{\text {delay }}(T S)_{U E(i)} \times \frac{p(T)_{U E(i)}}{A v g_{\text {Throughput }} U E(i)}\right.$

when $\mathrm{i} \leftarrow$ non-HARQ users and $\mathrm{p}(\mathrm{T})<T_{\text {bits }}(D L)_{U E(i)}$

Problem condition-3: Subjected to $n U E$ :

$\operatorname{ArgMax}\left\{\phi(i) \times \exp \left(\frac{b_{d} \times k}{b_{d}-p k t_{\text {delay }}(T s)_{U E(i)}}\right) \times \frac{p(T)_{U E(i)}}{A v g_{\text {Throughput }} U E(i)}\right.$

when $i \in$ both non-HARQ and HARQ users

Here $k$ is constant and $p(T)$ represent the cumulative number of bits in the buffer of UE(i) at time-slot (Ts) 1 or 2 .

The study designed an analytical algorithm approach for scheduling packets during LTE down-link transmission with simplified execution steps to address this problem.

Algorithm for down-link packet scheduling for LTE using proposed HARQ-S

Input parameters- nUE, $\mathrm{nRB}, \mathrm{Ts}, \mathrm{B}, \mathrm{P}, \mathrm{HT}=[\mathrm{HARQ}-1$, HARQ-2]

Output parameters- resource-aware pkt scheduling in downlink transmission

\section{Start:}

1. Initialize: nUE, nRB, Ts, B, P, HT

2. Deploy $\rightarrow$ UE 1, UE2 ........ UEn within the region of $\mathbf{L}$ $\mathbf{x ~ B}$

3. Enable proximity of deployment check: using eq. (1)

$$
U E_{x} \& U E_{y}=\sum_{k=1}^{U E_{t o t}} f\left(U_{k}\right) .
$$

4. Compute Euclidian distance for localization vector (L) computation

$$
D U E_{i j}=\sqrt{\sum\left[\left(U i j x^{2}\right),\left(U i j y^{2}\right)\right]} .
$$

5. Perform connectivity of UE 1 , UE2 UEn with eNodeB within particular cell $\mathrm{j}$.

7. Enable HARQ-S based scheduling:

8. Compute total bandwidth

$$
B=\sum_{i=1}^{N}\left(N_{s b}\right) i \ldots e q(2)
$$

9. eNodeB checks CQI of each UE(i)

10 Formulate the optimization problem:

To Maximize $\rightarrow n U E$ with fairness index $\left(\mathbf{f}_{\mathrm{i}}\right)$

Subjected to $A v g_{\text {Throughput }} U E(i)$ within

buffered lesser delay $\left(b_{d}\right)$

11. To solve this approach using HARQ-S:

12. if $(\mathrm{CQI}=$ High and Justifiable):

Allocate RBs $\rightarrow$ UE(i)s in Ts(1)

Compute

$n U E=\operatorname{ArgMax}\left\{\phi(i) \times p k t_{\text {delay }}(T S)_{U E(i)} \times \frac{T_{\text {bits }}(D L)_{U E(i)}}{A v g_{\text {Throughput }} U E(i)}\right.$

Use problem-condition-1: to compute nUE

if $\mathbf{p}(\mathbf{T})>T_{b i t s}(D L)_{U E(i)}$ for non-HARQ users

Use problem-condition-2: to compute nUE

if $\mathbf{p}(\mathbf{T})<T_{b i t s}(D L)_{U E(i)}$ for non-HARQ users

Use problem-condition-3: to compute nUE

if $i \in$ both non-HARQ and HARQ users

14.

Check pkt error using FEC and ARR

15. End

16. Elseif $($ Check priority $=\operatorname{Req}(\mathrm{pkt}))$

17. Use HARQ1/HARQ2 for scheduling the pkt in $\mathrm{Ts}(2)$ and allocate $\mathrm{RBs}$ to required UE(i)s

Check pkt error using FEC and ARR

18. Else

19. Schedule to next time slot UEs

20. End

21. Compute $A v g_{\text {Throughput }} U E(i), p k t_{\text {delay }}(T S)_{U E(i)}$

End

The proposed downlink packet scheduling combinedly uses the CQI, HARQ, and eNodeB scheduler's strength factors to effectively allocate the RBs to the justified UEs within the LTE network. Initially, the system deploys the eNodeBs and UEs with the optimal mode of deployment so that UEs that belong to a particular cell remains within the proximity of the region of LxB. Further, it can be seen that the scheduling algorithm is designed for both HARQ and non-HARQ UEs. Here the Scheduler operates with eNodeB, which maintains a buffer where pkts wait in the queue for re-transmission to the respective HARQ UEs in downlink and if the RBs are allocated. On the other hand, for non-HARQ users, pkts in eNodeB waits for initial transmission. As highlighted in the above algorithm, the HARQ-S employs both priority-based scheduling and scheduling of pkt based on the CQI computed value. The system allocates RBs to both HARQ and nonHARQ UEs. And similarly, the TBs are correctly received. The throughput and delay calculation is taken place considering the following mathematical expressions. 
$U E_{\text {throughput }}=1 / \mathrm{nUE} 1 / \mathrm{Ts} \sum \sum A v g_{\text {Throughput }} U E(i)_{\mathrm{Ts}}$

Here $1 \leq \mathrm{t} \leq \mathrm{Ts}$

Here $1 \leq \mathrm{i} \leq \mathrm{nUE}$

Average queuing delay $\mathrm{Q}_{\text {delay }}=1 / \mathrm{Ts} \sum 1 / \mathrm{nUE} \sum$

$p k t_{\text {delay }}(T S)_{U E(i)}$

Here $1 \leq \mathrm{t} \leq \mathrm{Ts}$

Here $1 \leq \mathrm{i} \leq \mathrm{nUE}$

The next section further discusses the experimental outcome obtained for different stimulation parameter settings within the framework design and execution environment scenario.

\section{RESULTS AND ANALYSIS}

As the proposed study is a benchmarking model, so the comparison is carried out among existing standard schedulers, e.g., round-robin, Best CQI, and HARQ scheduling. To validate the system model and its benchmarking with the existing RRS and BCQI-S, various combinations of the parameters are taken into consideration, which is having theoretical significance. The simulation parameter combinations are listed in Table V.

The typical performance graph for both HARQ-Type 1 and HARQ-Type-2 is discussed below, wherein both cases the number of resource blocks(nRB), time slot(T), number of user equipment(nUE), system bandwidth(B), and the base station transmit power $(\mathrm{P})$ is taken as $6,2,25,1.4 \mathrm{Mhz}, 23 \mathrm{dBm}$ respectively.

\section{A. Performance Graphs of throughput, Fairness, and Delay for HARQ Type I}

Fig. 6 illustrates the comparison of the HARQ-scheduling for HARQ type-1 with the RR-S and BCQI-S for Network throughput Vs. Base Station (BS) transmit power.

The throughput pattern increases for all three schedulers with an increment of the base station transmit power. The best CQI-S provides better throughput than the RR-S, whereas the presented concept of HARQ-scheduling yields superior outcomes compared to both BCQI-S and RR-S. The maximum value of network throughput for HARQ-scheduling appeared at the point of $1.8 \mathrm{~b} / \mathrm{s} / \mathrm{Hz}$ for the specific BS transmit power, 45 $\mathrm{dBm}$. On the other, in both BCQI-S and RR-S, when the increased transmit power is set to $45 \mathrm{dBm}$, then approximately a maximum of $1.7 \mathrm{~b} / \mathrm{s} / \mathrm{Hz}$ and $1.6 \mathrm{~b} / \mathrm{s} / \mathrm{Hz}$ network throughputs are obtained.

Fig. 7 illustrates the comparison of the HARQ-scheduling for HARQ type-1 with the RR-S and BCQI-S for Throughput vs. User's average SNR (dB).

The interpretation illustrates that similarly, in this experimental approach, it is observed that the trend of the curves for Network throughput (b/s/Hz) corresponds to 1. RR$\mathrm{S}, 2$. BCQI-S, and 3. HARQ-scheduling is progressively increasing for the incremented values ranges between -10 and $35(\mathrm{~dB})$. However, a closer analysis reveals that for a variable range of average users SNR (dB), BCQI-S outperforms RR-S in terms of throughput but at that same time found not much superior as compared to the presented HARQ-scheduling approach. HARQ-scheduling in every case of average user SNR $(\mathrm{dB})$ yields superior outcome, and at average users SNR value 35 , it produces global maximum value of network throughput, which is approximately $3.9(\mathrm{~b} / \mathrm{s} / \mathrm{Hz})$.

Fig. 8 compares the HARQ-scheduling for HARQ type-1 with the RR-S and BCQI-S for Network fairness index Vs. User's average SNR (dB).

TABLE V. SIMULATION PARAMETERS RANGE

\begin{tabular}{|l|l|l|l|}
\hline Sl. No & System Variable & Symbol & Range \\
\hline 1 & Number of user's equipment & nUE & $0,5,10,15,20,25$ \\
\hline 2 & Number of Resource Block & nRB & $6,15,25,50,75,100$ \\
\hline 3 & Time Slots & Ts & 2 \\
\hline 4 & System Bandwidth & B (Mhz) & $1.4,3,5,10,15,20$ \\
\hline 5 & Transmit Power & P & $\begin{array}{l}\text { Reference Power } \\
(23 \mathrm{dbm})\end{array}$ \\
\hline 6 & HARQ-Type & HT & \\
\hline
\end{tabular}

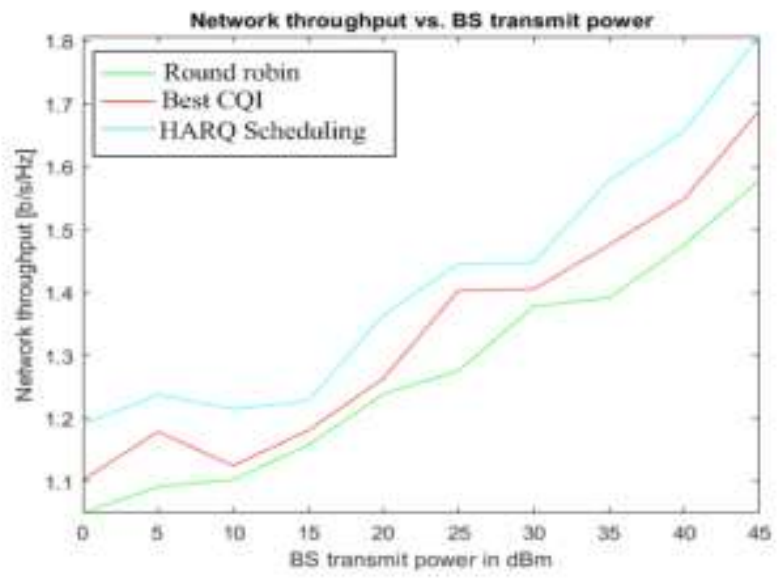

Fig. 6. Network Throughput (bits/Hz) Vs. Base Station Transmit Power.

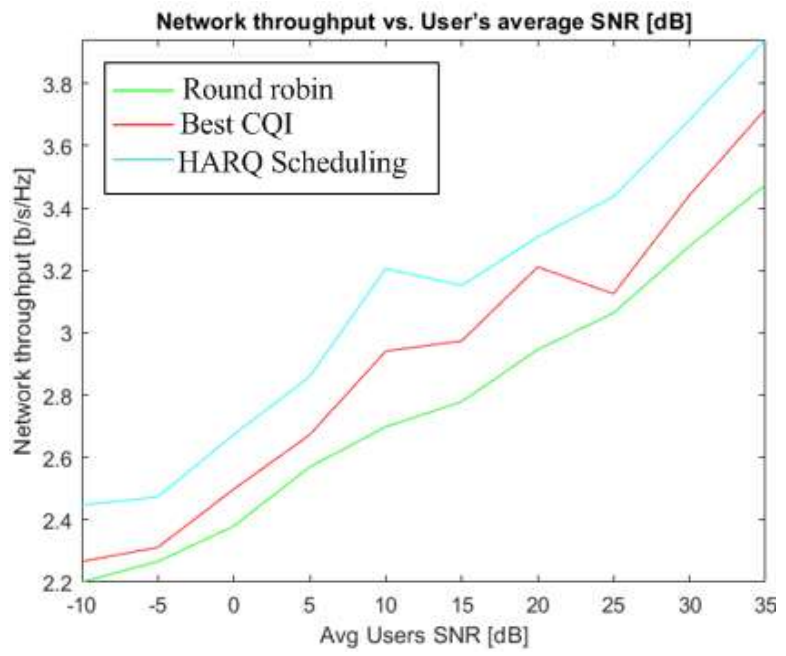

Fig. 7. Network Throughput (bits/Hz) Vs. Average users SNR (dB). 


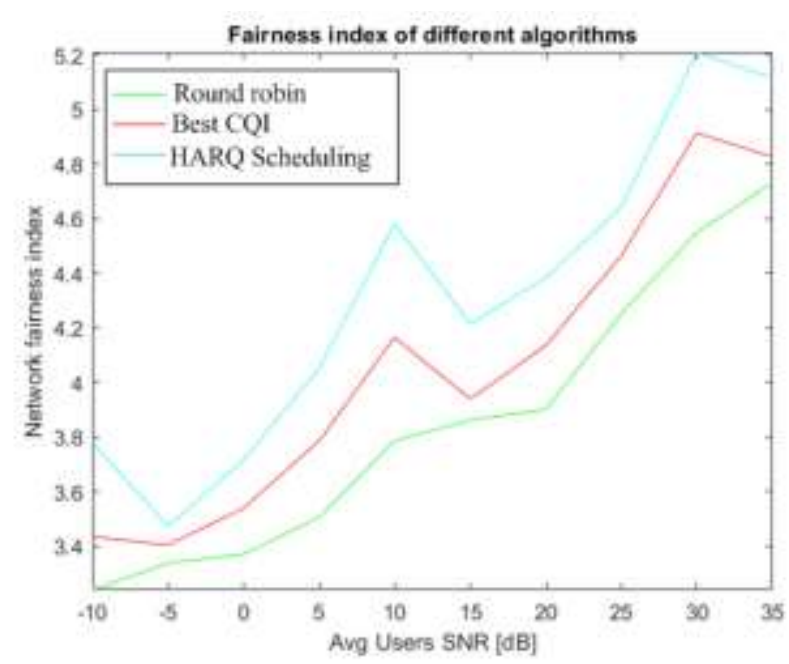

Fig. 8. Network Fairness Index vs. Average users SNR (dB).

The analysis of the network fairness index from the validation of these algorithms such as as: 1) RR-S, 2) BCQI-S, and 3) HARQ-scheduling shows that in this testing scenario, the network fairness index movement trend is quite higher in HARQ-scheduling in contrast with BCQI-S and RR-S. But if we consider the case of both BCQI-s and RR-S, then the BCQI-S outcome for the network fairness index must dominate more as compared to the outcome corresponding to RR-S. The maximum value of network index fairness is obtained in HARQ-scheduling, which is at 5.2 for $30(\mathrm{~dB})$ average users SNR.

Fig. 9 illustrates the comparison of the HARQ-scheduling for HARQ type-1 with the RR-S and BCQI-S for Delay Vs. BS transmit power in $\mathrm{dBm}$.

With increasing BS transmit power in $\mathrm{dBm}$, it is observed that the amount of Delay computed in the case of RR-S is quite higher, and the impact remains till the value of approximately 1.9 when the BS transmit power is set to $45 \mathrm{dBm}$. On the other hand, in both the cases of BCQI-S and HARQ-scheduling, the trend of the delay curve movement is found increasing but observed lesser as compared to RR-S. Out of all these three approaches, HARQ-scheduling exhibits consistent and lesser Delay with increasing BS transmit power.

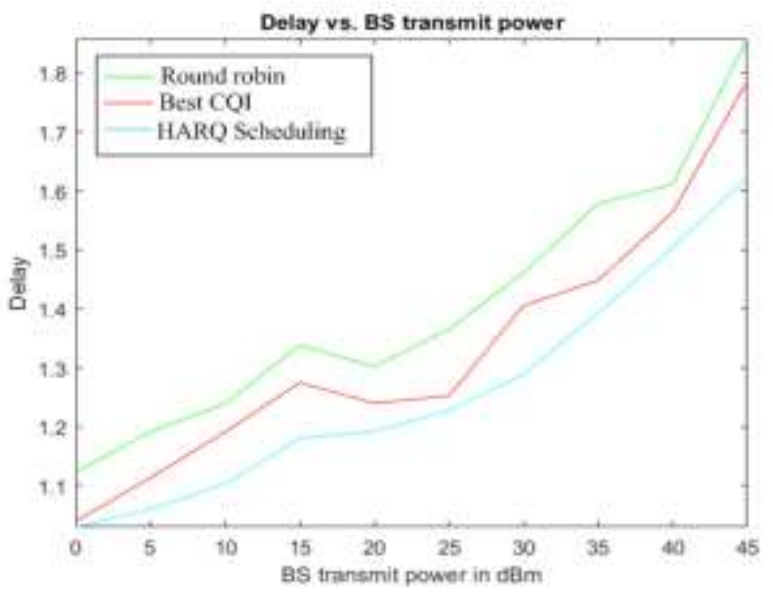

Fig. 9. Delay vs. BS Transmit Power in $\mathrm{dBm}$.
Fig. 10 illustrates the comparison of the HARQ-scheduling for HARQ type-1 with the RR-S and BCQI-S for Delay vs. User's average SNR (dB).

The closer interpretation of the delay analysis outcome is again assessed for the user's average SNR (dB). Here, the pattern of the curve of the delayed movement in each case of 1 . RR-S, 2. BCQI-S, and 3. HARQ-scheduling is increasing progressively, but the values of the outcome for Delay in the case of RR-S get negatively influenced when the average user's SNR ranges between -10 to $35(\mathrm{~dB})$. In this case, also when the delayed outcome is concerned- HARQ-scheduling outperforms the other approaches and significantly minimizes the Delay up to the approximate value of 3.67778 .

Fig. 11 illustrates the comparison of the HARQ-scheduling for HARQ type-1 with the RR-S and BCQI-S for Delay of different algorithms vs. User's average SNR (dB).

The Delay analysis in this experimental approach considers the execution of algorithms from the time complexity viewpoint. It clearly outlines that HARQ-scheduling imposes optimized steps of execution. It leads to a scenario where, with an increasing number of average users SNR, the Delay found bounded within a maximum approximate value of 5.4587. In both RR-S and BCQI-S, the delay computation yields an outcome that is quite higher than the presented approach of HARQ-scheduling for increasing value of average users SNR $(\mathrm{dB})$.

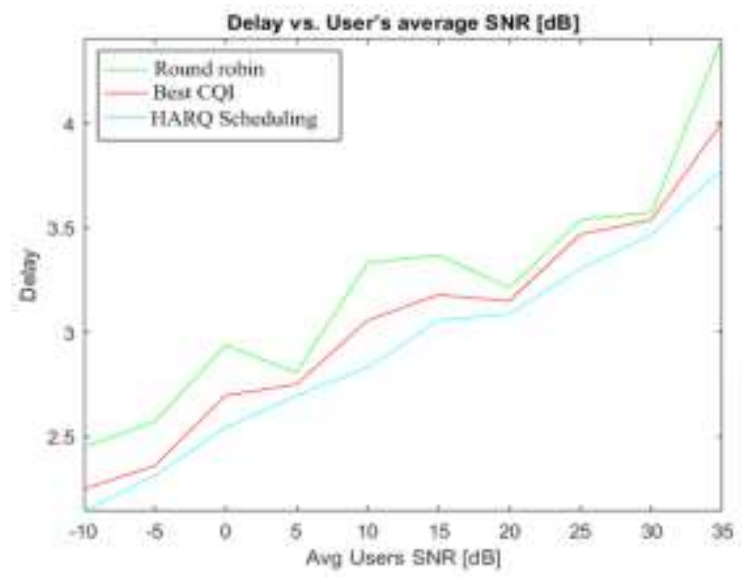

Fig. 10. Delay vs. Average users SNR (dB).

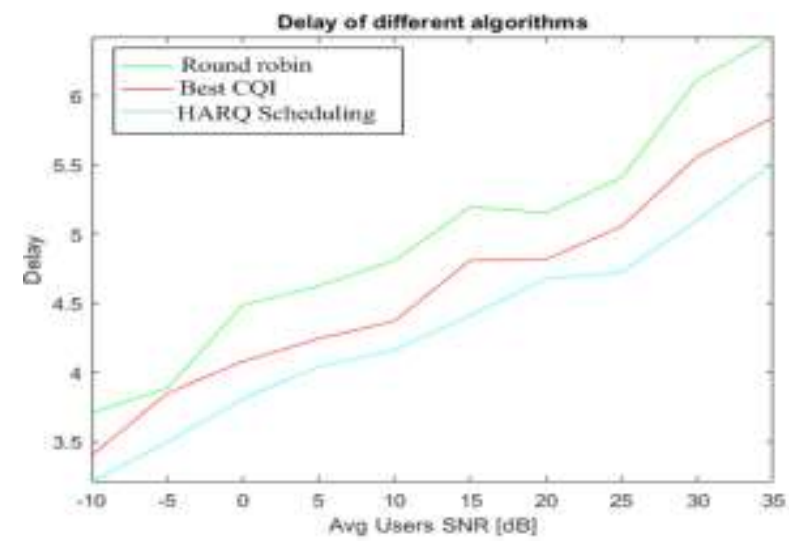

Fig. 11. Delay of different Algorithms vs. Average users SNR (dB). 


\section{B. Performance Graphs of throughput, Fairness, and Delay for HARQ Type II}

Fig. 12 illustrates the comparison of the HARQ-scheduling for HARQ type-2 with the RR-S and BCQI-S for Network throughput Vs. BS transmit power in $\mathrm{dBm}$.

The analysis of network throughput $(\mathrm{b} / \mathrm{s} / \mathrm{Hz})$ for HARQ type-2 shows that in comparison with RR-S and BCQI-S, the HARQ-scheduling approach accomplishes superior outcomes increasing values of $\mathrm{BS}$ transmit power in $\mathrm{dBm}$, which ranges between 0 to 45 . If we consider the cases of RR-S and BCQI-S, then it can be observed that BCQI-S attain better network throughput as compared to RR-S. The maximum value of throughput in the case of HARQ-scheduling appeared at point 2.1 for the BS transmit power of $45 \mathrm{dBm}$.

Fig. 13 illustrates the comparison of the HARQ-scheduling for HARQ type-2 with the RR-S and BCQI-S for Network throughput Vs. User's average SNR (dBm).

Another experimental approach is assessed to evaluate the network throughput $(\mathrm{b} / \mathrm{s} / \mathrm{Hz})$ with the increasing amount of Average users SNR $(\mathrm{dB})$ ranges between -10 and 35. The visual representation of the comparable outcome shows thatSimilarly to the above cases, which is illustrated in Fig. 12, HARQ-scheduling accomplishes superior outcome for the increased values of average users SNR (dB). The visual data representation of the network throughput curves for RR-S and BCQI-S also shows that in contrast with RR-S, the approach of BCQI-S attain a superior outcome where the maximum value of throughput appears at approximately $3.6 \mathrm{~b} / \mathrm{s} / \mathrm{Hz}$. Fig. 14 illustrates the comparison of the HARQ-scheduling for HARQ type-2 with the RR-S and BCQI-S for Network fairness index Vs. User's average SNR (dBm).

The computed outcome analysis corresponding to the network fairness index for these three algorithms also shows that that index movement is significantly higher and progressive upwards in the case of HARQ-scheduling compared to RR-S and BCQI-S algorithms. For each value of average users SNR, which ranges between -10 and 35, the outcome found superior in HARQ-scheduling and marginally differs from BCQI-S. However, among all these three algorithms, RR-S's performance is quite lower when the parameter network fairness index is concerned with increasing values of average users SNR $(\mathrm{dB})$.

Fig. 15 illustrates the comparison of the HARQ-scheduling for HARQ type-2 with the RR-S and BCQI-S for Delay vs. BS transmit power in $\mathrm{dBm}$.

The Delay's quantified outcome using three different algorithms, such as RR-S, BCQI-S, and the formulated approach of HARQ-scheduling, are visually represented in Fig. 15. It clearly shows that Delay's quantified outcome is significantly lesser in the case of HARQ-scheduling even though the increasing BS transmit power in $\mathrm{dBm}$ increases from 0 to 45. The outcome of the HARQ-scheduling in Delay's context is found a maximum of 1.9786, which is significantly lesser than the other baseline scheduling models.

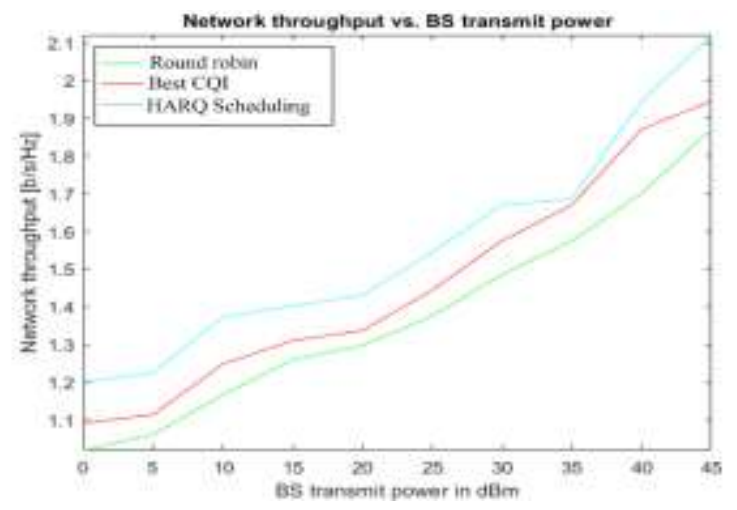

Fig. 12. Network throughput $(\mathrm{b} / \mathrm{s} / \mathrm{Hz})$ vs. BS Transmit Power in $\mathrm{dBm}$.

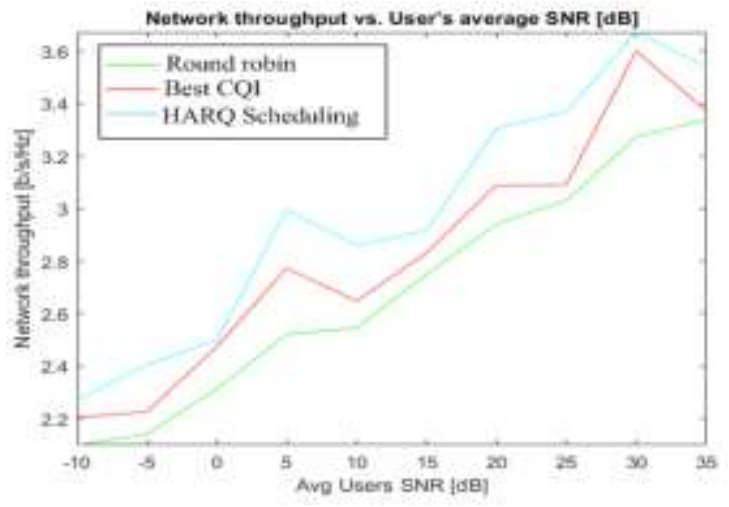

Fig. 13. Network throughput (b/s/Hz) vs. user's Average SNR (dBm).

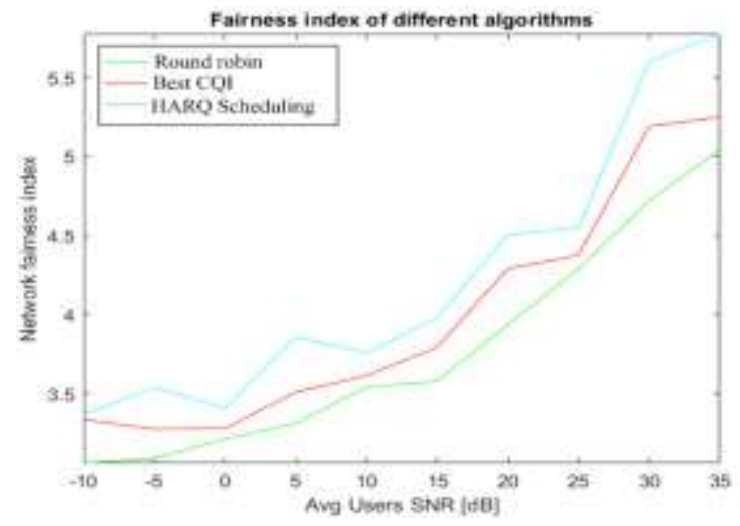

Fig. 14. Extended Analysis of Network Fairness Index.

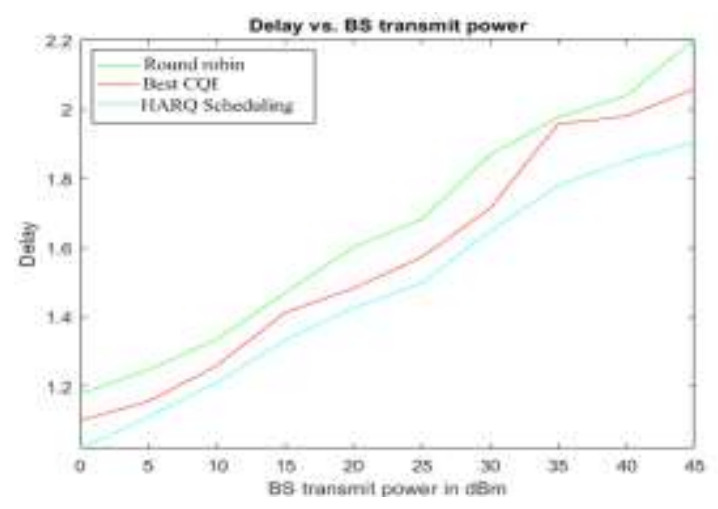

Fig. 15. Extended Analysis of Delay. 
Fig. 16 illustrates the comparison of the HARQ-scheduling for HARQ type-2 with the RR-S and BCQI-S for Delay vs. Average users SNR (dB).

The delay performance analysis, in this case, was observed concerning the increased values of Average users SNR (dB), which ranges between -10 and 35 . For all three approaches, it is observed that the trend of the delay movement curve increases with the values of average users SNR $(\mathrm{dB})$. However, the BCQI-S attains superior outcome in the context of Delay as compared to RR-S. Still, let's consider the overall performance of the Delay. HARQ-scheduling is superior as it yields a significantly lesser Delay even though the $\%$ of the average user's SNR is increased.

Fig. 17 illustrates the comparison of the HARQ-scheduling for HARQ type-2 with the RR-S and BCQI-S for the Delay of different algorithms Vs. Average users SNR (dB).

The delayed outcome here indicates the algorithm performance from a time complexity viewpoint. A closer interpretation reveals that in the presented approach of HARQScheduling, the curve of Delay's curve progressively increases concerning the increasing data points of SNR $(\mathrm{dB})$. Still, if we compare the performance of HARQ-scheduling with RR-S and BCQI-S, then it can be observed that HARQ-scheduling exhibits a superior performance graph among all of these.

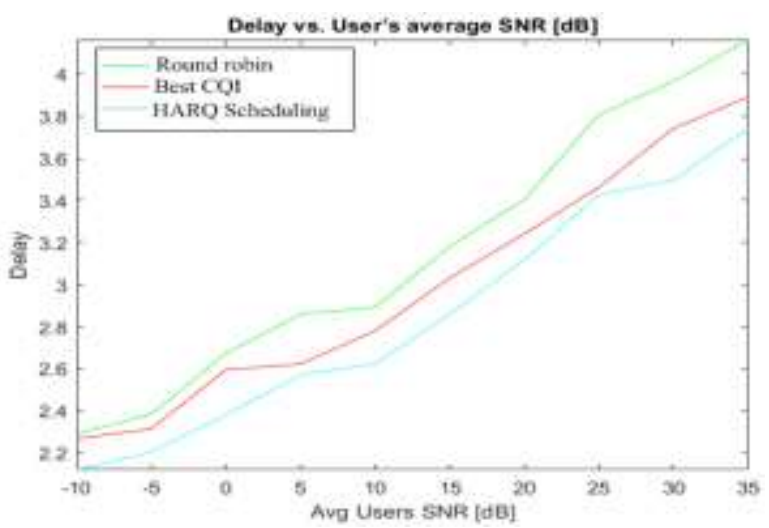

Fig. 16. Analysis of the Impact of SNR on Delay.

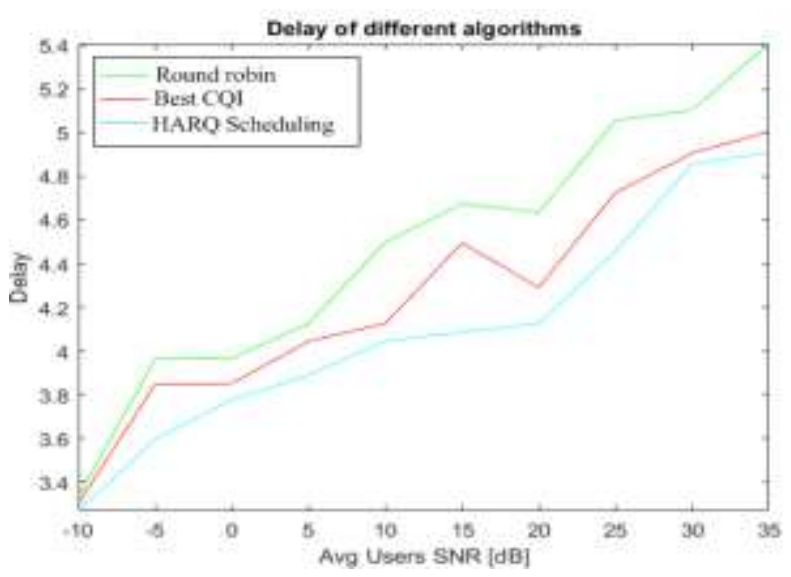

Fig. 17. Delay of different Algorithms vs. Average users SNR (dB).

\section{CONCLUSION}

The study outcome of the proposed study shows that the HARQ scheduler offers approximately $37 \%$ improvement from Round Robin and approximately $26 \%$ improvement from the best CQI algorithm concerning its network throughput in the presence of variable transmit power of the base station. The patterns of network throughput remain nearly the same when evaluated with increasing SNR. HARQ scheduler is also witnessed to offer approximately $40 \%$ of reduction in delay from Best CQI and approximately $43 \%$ of reduction from Round-robin in the presence of variable transmit power and different SNR values. Similar outcomes are also observed for HARQ type-II algorithms.

Scheduler optimization for balancing the throughput, fairness, and optimal resource allocation is a very active research problem study and significant in the growing UEs density. In this paper, an essential of the general requirements, physical layer characteristics, and the research trend in the scheduling model for LTE /NR is studied, directly impacting the system performance. The OFDM enabled eNodeB to divide the bandwidth into resource element, allocated to the UEs by the schedulers in eNodeB or Base stations (BS). The proposed HARD-S handles the trade-off issues between the Best CQI and Round Robin to balance optimal throughput and fairness. The time -slot servicing for the RB block takes place on priority dependencies of CQI and HARQ to balance the tradeoff between the throughput and the fairness. The benchmarking of the HARQ-S is done with the Best-CQI and Round robin for throughput and fairness and observe optimization in the throughput compared to the RRS and fairness optimization compared to the BCQI. A better scheme of OFDM-MIMO may further claim to have better throughput.

The future work of the proposed study will be to achieving better form of computational efficiency. The present work offers a benchmarking testbed to prove HARQ is the best controller where a distinct section of cumulative memory of HARQ is allocated to each carrier. However, there is a possibility of more increase of incoming HARQ requests by optimizing the computational efficiency. This will be investigated in the upcoming work direction where $5 \mathrm{G}$ scenarios can be considered with more approximation cases to leverage throughput.

\section{REFERENCES}

[1] Parameswaran V., Shanmugasundaram M, "Journey of Wireless Communication. In: Ranganathan G., Chen J., Rocha Á. (eds) Inventive Communication and Computational Technologies. Lecture Notes in Networks and Systems, Vol 89. Springer, Singapore, 2020.

[2] Holma H, Lunttila T. LTE-Advanced Evolution. 5 G Technology: 3 GPP New Radio. 2020 Feb 10:477-99.

[3] I. Pospishny, V. Vasyuk, S. Romanchyk, O. Dovzhenko, O. Dony and V. Shvaichenko, "3GPP long term evolution (LTE)," International Conference on Modern Problems of Radio Engineering, Telecommunications and Computer Science (TCSET), Lviv-Slavske, pp. 192-192, 2010.

[4] R. Cai, X. Wang, Y. Wang, S. Xiao, C. Wang, and W. Wang, "A Novel and Effective User Scheduling Scheme for Heterogeneous Scenario in LTE-A System," IEEE International Conference on Computer and Information Technology, Xi'an, 2014, pp. 679-683. 
[5] R. B. Abreu, G. Pocovi, T. H. Jacobsen, M. Centenaro, K. I. Pedersen and T. E. Kolding, "Scheduling Enhancements and Performance Evaluation of Downlink 5G Time-Sensitive Communications," in IEEE Access, vol. 8, pp. 128106-128115, 2020, doi: 10.1109/ACCESS.2020.3008598.

[6] G. Femenias, F. Riera-Palou, X. Mestre and J. J. Olmos, "Downlink Scheduling and Resource Allocation for 5G MIMO-Multicarrier: OFDM vs. FBMC/OQAM," in IEEE Access, vol. 5, pp. 13770-13786, 2017, 10.1109/ACCESS.2017.2729599.

[7] J. Liang, C. Hsu, J. Chen, K. Wu, and Y. Tseng, "Three-Stage DRX Scheduling for Joint Downlink Transmission in C-RAN," in IEEE Wireless Communications Letters, vol. 9, no. 2, pp. 129-133, Feb. 2020, doi: 10.1109/LWC.2019.2943471.

[8] D. A. Omer, A B. N. Mustafa, "LTE FDD vs. LTE TDD from a QoS Perspective," Journal of Electronics and Communication Engineering (IOSR-JECE), Vol. 10, Issue. 2, pp. 96-100, 2015.

[9] Xylomenos, George \& Vogkas, Vasilis \& Thanos, George. (2008). The multimedia broadcast/multicast service. Wireless Communications and Mobile Computing. 8. 255-265. 10.1002/wcm. 463.

[10] U. Hani and K. K. Samota, "Particle Swarm Optimization Algorithm to Improve Access Delay in 5G Technology," 2018 Second International Conference on Advances in Computing, Control and Communication Technology (IAC3T), Allahabad, India, 2018, pp. 23-27.

[11] A. Saxena and R. Sindal, "Performance Analysis of MAC Scheduler in LTE (EUTRAN) for "ASAR": Resource Allocation," 2016 10th International Conference on Next Generation Mobile Applications, Security and Technologies (NGMAST), Cardiff, 2016, pp. 6-11.

[12] M. Minelli, M. Ma, M. Coupechoux and P. Godlewski, "Scheduling Impact on the Performance of Relay-Enhanced LTE-A Networks," in IEEE Transactions on Vehicular Technology, vol. 65, no. 4, pp. 24962508, April 2016.

[13] T. K. Ramesh, "A Survey on scheduling algorithm for downlink in LTE cellular network," 2019 International Conference on Smart Systems and Inventive Technology (ICSSIT), Tirunelveli, India, 2019, pp. 219-223.

[14] A. Chilmulwar and V. Sinha, "A Novel Machine Learning-Based MAC Scheduler Algorithm using ARIMA," 2019 16th IEEE Annual Consumer Communications \& Networking Conference (CCNC), Las Vegas, NV, USA, 2019, pp. 1-8.

[15] J. O. Olaifa and D. Arifler, "Using system-level simulation to evaluate downlink throughput performance in LTE-A networks with clustered user deployments," 2016 1st International Workshop on Link- and System Level Simulations (IWSLS), Vienna, 2016, pp. 1-6.

[16] S. Chaudhuri, I. Baig, and D. Das, "QoS aware downlink scheduler for a carrier aggregation LTE-Advance network with efficient carrier power control," 2016 IEEE Annual India Conference (INDICON), Bangalore, 2016, pp. 1-6.

[17] N. D. Adesh and A. Renuka, "Impact of traffic burst on the behavior of TCP variants for different LTE downlink schedulers," 2017 3rd International Conference on Applied and Theoretical Computing and Communication Technology (iCATccT), Tumkur, 2017, pp. 18-23.

[18] K. G. Sundari, R. Gunasundari and K. Jayanthi, "Prioritized dynamic multi traffic scheduler for downlink in LTE," 2015 2nd International Conference on Electronics and Communication Systems (ICECS), Coimbatore, 2015, pp. 1469-1473.
[19] B. Rebekka, B. V. Kumar and B. Malarkodi, "Radio resource allocation with energy efficiency-throughput balancing for LTE downlink," 2015 2nd International Conference on Electronics and Communication Systems (ICECS), Coimbatore, 2015, pp. 111-115. doi: 10.1109/ECS.2015.7124754.

[20] Ö. Yildiz and R. Sokullu, "A novel mobility aware downlink scheduling algorithm for LTE-A networks," 2017 Ninth International Conference on Ubiquitous and Future Networks (ICUFN), Milan, 2017, pp. 300305.

[21] A. Hayuwidya, M. E. Ernawan, and Iskandar, "Scheduling techniques in release 8 LTE network," 2017 11th International Conference on Telecommunication Systems Services and Applications (TSSA), Lombok, 2017, pp. 1-5.

[22] R. E. Ahmed and H. M. AlMuhallabi, "Throughput-fairness trade-off in LTE uplink scheduling algorithms," 2016 International Conference on Industrial Informatics and Computer Systems (CIICS), Sharjah, 2016, pp. 1-4.

[23] M. O. Kayali, Z. Shmeiss, H. Safa and W. El-Hajj, "Downlink Scheduling in LTE: Challenges, improvement, and analysis," 2017 13th International Wireless Communications and Mobile Computing Conference (IWCMC), Valencia, 2017, pp. 323-328. doi: 10.1109/IWCMC.2017.7986307.

[24] A. Hajjawi, M. Ismail, N. F. Abdullah, M. N. Hindia, A. M. AlSamman, and E. Hanafi, "Investigation of the impact of different scheduling algorithm for Macro-Femto-Cells over LTE-A networks," 2016 IEEE 3rd International Symposium on Telecommunication Technologies (ISTT), Kuala Lumpur, 2016, pp. 125-128.doi: 10.1109/ISTT.2016.7918098.

[25] A. A. A. Rahman, A. Man, A. K. Samingan, C. Y. Yeoh and I. Suleiman, "Fair boundary scheduler for LTE system," 2016 IEEE Symposium on Computer Applications \& Industrial Electronics (ISCAIE), Batu Feringghi, 2016, pp. 11-15.doi: 10.1109/ISCAIE.2016.7575028.

[26] K. G. Sundari, K. Jayanthi, and R. Gunasundari, "Prioritized dynamic multi traffic scheduler for downlink in LTE," 2015 2nd International Conference on Electronics and Communication Systems (ICECS), Coimbatore, 2015, pp. 1546-1550. doi: 10.1109/ECS.2015.7124847.

[27] S. Alotaibi and R. Akl, "Packet scheduling bandwidth type-based mechanism for LTE," 2017 IEEE 8th Annual Ubiquitous Computing, Electronics and Mobile Communication Conference (UEMCON), New York, NY, 2017, pp. 363-368.

[28] S. Harkusha, O. Harkusha, and V. Olkhovsky, "Result research model of scheduling block allocation in downlink LTE," 2016 Third International Scientific-Practical Conference Problems of Infocommunications Science and Technology (PIC S\&T), Kharkiv, 2016, pp. 92-95. doi: 10.1109/INFOCOMMST.2016.7905345.

[29] M. Bechir Dadi and R. Belgacem Chibani, "Scheduling performance's study for LTE downlink system," International Conference on Green Energy Conversion Systems (GECS), Hammamet, 2017, pp. 1-4. doi: 10.1109/GECS.2017.8066174.

[30] A. B. Shams, S. R. Abied and M. A. Hoque, "Impact of user mobility on the performance of downlink resource scheduling in Heterogeneous LTE cellular networks," 3rd International Conference on Electrical Engineering and Information Communication Technology (ICEEICT), Dhaka, 2016, pp. 1-6.doi: 10.1109/CEEICT.2016.7873091. 